9 - ORIGINAL ARTICLE WOUND HEALING

\title{
Use of insulin-like growth factor in the healing of open wounds in diabetic and non-diabetic rats ${ }^{1}$
}

\author{
Rosi Aparecida Nunes Achar ${ }^{\mathrm{I}}$, Thiago Couto Silva ${ }^{\mathrm{II}}$, Eduardo Achar ${ }^{\mathrm{III}}$, Roosecelis Brasil Martines ${ }^{\mathrm{IV}}$, José Lucio Martins Machado ${ }^{\mathrm{V}}$ \\ IFellow Master degree, Postgraduate Program in Health Sciences, Sao Paulo State Public Servant Hospital (IAMSPE). Associate Professor, Experimental \\ Surgery, Sao Paulo City University (UNICID), Sao Paulo-SP, Brazil. Conception, design, scientific and intellectual content of the study; manuscript writing. \\ II Graduate student, Medical School, UNICID, Grant from Institutional Program for Scientific Initiation (PIBIC) of the National Council of Technological \\ and Scientific Development (CNPq), Ministry of Science, Technology and Inovation, Sao Paulo-SP, Brazil. Acquisition of data, technical procedures. \\ IIIPhD, Associate Professor, Experimental Surgery, UNICID, Sao Paulo-SP, Brazil. Technical procedures, critical revision. \\ ${ }^{\text {Iv }} \mathrm{PhD}$, Medical Pathologist, Adolfo Lutz Institute, Sao Paulo-SP, Brazil. Histological analysis. \\ ${ }^{v} \mathrm{PhD}$, Associate Professor, Postgraduate Program in Health Sciences, IAMSPE. Associate Professor, Experimental Surgery, UNICID, Sao Paulo-SP, \\ Brazil. Manuscript writing, supervised all phases of the study.
}

\section{ABSTRACT}

PURPOSE: To analyze the effects of application of $1 \%$ and $3 \%$ insulin-like growth factor I (IGF-1) cream on the process of wound healing in induced skin lesions in diabetic and non-diabetic rats and evaluate its effect on expression of myofibroblasts.

METHODS: Ninety-six Wistar adult male rats were divided into six groups, with 16 rats in each group, as follows: group 1: nondiabetic, untreated; group 2: non-diabetic, treated with 1\% IGF-1 cream; group 3: non-diabetic, treated with 3\% IGF-1 cream; group 4: diabetic, untreated; group 5: diabetic, treated with 1\% IGF-1 cream; and group 6: diabetic, treated with 3\% IGF-1 cream. In groups 4,5 , and 6 , diabetes was induced by intravenous injection of alloxan. After diabetes had been induced, animals were mantained for 3 months. The experimental procedure consisted of the creation of a circular incision of $0.9 \mathrm{~mm}$ in diameter using a metal punch. Following this, wounds were treated daily according to the assigned treatment regimen. Groups 2 and 5 were treated with $1 \%$ IGF-1 cream, groups 3 and 6 with 3\% IGF-1 cream, and groups 1 and 4 and the untreated groups with 0.9\% saline solution. From each group, samples from 4 rats were taken at three, seven, 14, and 21 days after the injury. Samples were fixed in 10\% formalin to prepare slides for histological analysis. Slides stained with hematoxylin-eosin (H\&E) and Masson were observed vascular proliferation, mononuclear cells, polymorphonuclear cells, fibroblast proliferation, re-epithelialization, and collagen fibers. This study analyzed the expression of $\alpha$-smooth muscle actin using specific antibodies to correlate the temporal expression of $\alpha$-smooth muscle-specific actin ( $\alpha$-SM actin), a molecular marker for myofibroblast transformation.

RESULTS: Macroscopic observation of wounds showed a more rapid re-epithelialization of wounds treated with IGF. Regarding acute inflammatory reactions, the results of the analysis of vascular proliferation and polymorphonuclear and mononuclear cells showed no statistically significant differences in any of the periods studied (according to the results of a Mann-Whitney test). The initial immunohistochemical analysis of tissue samples conducted to compare the expression of $\alpha$-smooth muscle actin between groups showed a relevant response in the expression of myofibroblasts. Data were analyzed using ANOVA and were found to be statistically significant. CONCLUSION: The topical application of $1 \%$ and $3 \%$ IGF-1 creams increases the expression of myofibroblasts in the process of wound healing in rats.

Key words: Insulin-Like Growth Factor I. Myofibroblasts. Diabetes Mellitus. Wound Healing. Alloxan. Rats. 


\section{Introduction}

Cytokines are small, serum-soluble proteins that interact with cell surface receptors. These interactions with specific transmembrane receptors activate a cascade of signals that result in a cellular response.

Insulin-like growth factor I (IGF-1; a growth factor similar to insulin) is a cytokine that participates in the cellular granulation process during wound healing. During healing, its expression is increased. However, diabetic patients show overall decreased levels of IGF-1 expression ${ }^{1}$. IGF-1 is a polypeptide consisting of 70 amino acids with a molecular weight of $7.47 \mathrm{kDa}$ with an amino acid sequence that is very similar to insulin. IGF-1 exerts similar effects on growth as insulin. The anabolic effects of IGF-1 include stimulation of DNA synthesis, cell proliferation, protein synthesis, and glucose transport.

Previous studies have shown a relationship between inflammation markers interleukin 6 (IL-6), interleukin 8 (IL-8), and IGF-1 and microvascular changes in patients with type 1 diabetes. These studies found that IGF-1 levels were decreased in the control group and that IL-6 and IL-8 levels were elevated in patients with type 1 diabetes ${ }^{2}$.

IGF-1 has also been described as an agent that acts anabolically and anti-catabolically in a wide variety of cell types ${ }^{3,4}$.

In the context of wound healing, the evidence suggests that IGF-1 is mitogenic for keratinocytes and that fibroblasts inhibit the process of apoptosis, thereby reducing the production of inflammatory cytokines and stimulating extracellular matrix production $^{5}$.

IGF-1 expression is modulated during wound healing. Expression is increased in wound fluid caused by burns and is also significantly increased during the healing process of skin cells, which ordinarily express only small amounts of this protein ${ }^{3,6}$. This supports the idea that successful healing is associated with IGF-1 levels.

During granulation, tissue fibroblasts develop various structural and biochemical characteristics similar to ultra-smooth muscle cells, including the presence of microfilaments and the expression of $\alpha$-smooth muscle actin. When the granulation tissue develops into a scar, the myofibroblasts containing $\alpha$-smooth muscle actin disappear, probably as a result of apoptosis.

The mechanisms leading to myofibroblast development have been investigated elsewhere ${ }^{7}$. Recent studies have reported the mitogenic action of myofibroblast differentiation and proliferation by IGF-1 ${ }^{8,9}$.

During the repair of normal tissues, the controlled activation of myofibroblasts contributes to the restoration of tissue integrity ${ }^{10}$. The most widely used marker for myofibroblast differentiation is $\alpha$-smooth muscle actin. Under normal conditions, fibroblast cells exhibit little or no short actin and, in the extracellular matrix produced by an injury, fibroblasts are activated by cytokines released locally from resident and inflammatory cells migrating into the damaged tissue to synthesize extracellular matrix components ${ }^{3}$.

The aim of this study was to evaluate whether the topical application of IGF-1 cream influences the tissue repair process of wounds in diabetic and non-diabetic rats. This evaluation was performed using immunohistochemical analysis of the expression of myofibroblasts via antibodies to $\alpha$-smooth muscle actin.

\section{Methods}

This research protocol was approved by the Ethics Committee on Animal Use (CEUA) City University of Sao Paulo, fulfilling Federal Law 11,794 and Decree No. 6.689 of July 15, 2009, which regulates Law 11.794. The animals were housed in a vivarium provided by the City University of São Paulo, where the macro-environment was controlled with a photoperiod of $12 \mathrm{~h}$.

\section{Animals and induction of diabetes}

Ninety-six adult male Wistar rats weighing 250 to $300 \mathrm{~g}$ were included in the study. Forty-eight animals were non-diabetic and 48 animals were diabetic. Diabetes mellitus (DM) was induced by intravenous administration of alloxan (5,6-dioxouracil monohydrate) with a single dose of $42 \mathrm{mg} / \mathrm{kg}$. Subsequently, the animals were maintained on normal food and water.

\section{Assessment of diabetes}

Glucose levels were measured on the 7th and 14th days after induction and were sampled from animals whose blood glucose levels were greater than $250 \mathrm{mg} / \mathrm{dl}$. The results of glucose levels included in the study on the day of induction varied from $69-102 \mathrm{mg} / \mathrm{dl}$ on the day 7 to $260-561 \mathrm{mg} / \mathrm{dl}$ on day 14 and were between $285-593 \mathrm{mg} / \mathrm{dl}$.

Plasma glucose levels were determined from blood samples collected from the caudal vein using a glucometer AccuChek Advantage II (Roche).

Glycated hemoglobin (HA1C) levels were used as a reliable indicator of chronic diabetes, as their high values indicate persistently high blood glucose levels, as in diabetes. [Remark 2] Blood was collected from the tail vein at the time of collection of 
material for histological analysis before euthanasia to demonstrate the persistence of high blood glucose throughout the experiment duration. Diabetic animals average HA1C levels of $10.88 \% \pm$ 1.048, whereas non-diabetic animals had an average HA1C levels of $5.12 \% \pm 0.463$.

Diabetic rats, in addition to having high levels of blood glucose and HA1C, also exhibited typical features of the disease, including polyuria, polyphagia, polydipsia, and severe weight loss.

The pathological manifestations of diabetes in the kidneys, retina, and blood vessels are related to metabolic abnormalities caused by the disease over time. Therefore, following the induction of diabetes with alloxan, it was necessary that the diabetic animals were kept for three months prior to the experiment, to give enough time for organic changes to be observed in this group ${ }^{11}$.

\section{Study groups}

The animals were divided into six groups of 16. Group 1 included non-diabetic rats receiving no treatment (NDNT), group 2 included in non-diabetic rats that treated with $1 \%$ IGF-1 cream (NDIGF1\%), group 3 included non-diabetic rats that were treated with 3\% IGF-1 cream (NDIGF3\%), group 4 included diabetic rats that received no treatment (DNT), group 5 included diabetic rats treated with $1 \%$ IGF-1 cream (DIGF1\%), and group 6 included rats treated with $3 \%$ IGF-1 cream (DIGF3\%).

\section{Anesthesia and surgical procedures}

Three months after induction of diabetes, all animals received general anesthesia via an intraperitoneal injection of ketamine hydrochloride (75 mg/kg Vetbrands, Jacarei-SP, Brazil) and xylazine $(10 \mathrm{mg} / \mathrm{kg}$ Vetbrands). After anesthesia, the dorsal region was shaved and antisepsis was performed over the shaved area with chlorhexidine gluconate $2 \%$, and using a autoclavesterilized razor-sharp metal punch, a $0.9-\mathrm{cm}$ fragment of was removed until exposure of the dorsal muscle fascia.

\section{Treatment of surgical wounds}

Following this, the animals were reconditioned in specific boxes, divided according to their respective groups, and rats in each group received their assigned treatment, with saline only being administered to the wounds of the animals in groups 1 and 4. This procedure was repeated daily for the duration of the experiment. All rats were examined daily for their mobility, food intake, and water consumption.

\section{Histological analysis}

On days $3,7,14$, and 21 after surgery, the animals were weighed and anesthetized in the same manner as for the initial surgery. The animals were then affixed to the operating table, and the wound was excised with a margin $1 \mathrm{~cm}$ of healthy skin around the lesion, incising deeply into the fascia muscle. This time, the tail vein was punctured and blood samples were taken for measurement of HA1C. Following collection of materials, the animals were sacrificed.

Each sample was individually identified, fixed in $10 \%$ formalin solution, and sent to the pathology laboratory where the slides were prepared for histological analysis.

The samples were embedded in paraffin, cut into transverse sections $0.4 \mathrm{~mm}$ thick using a microtome, and stained with hematoxylin-eosin (H\&E) for comprehensive assessment of tissue sections, and by Masson's Trichrome (TM) to assess the presence of collagen fibers.

Histological analysis was performed by the same pathologist who was blinded to the identity of the groups. H\&Estained samples were classified according to the intensity that would have been found and has been attributed to the index of histological findings.

The sections stained with H\&E were scored according to the following scale: 0 (absent), 1 (mild), 2 (moderate), and 3 (severe). In each case, we analyzed the epidermis, the superficial dermis, and the deep dermis. Epidermal histological parameters were used to evaluate whether there was presence of ulcers, crusts or epithelialization. The dermis was evaluated for the presence of congestion, hemorrhage, necrosis, vascular proliferation, spindle cells suggestive of myofibroblasts, scar retraction, and pigments.

The dermal inflammatory infiltrate was evaluated for its intensity and the cellular elements present.

Sections stained using Masson's Tricromic technique were used to evaluate the presence of collagen fibers in the wound, scored according to the following scale: 0 (absent), 1 (mild), 2 (moderate), and 3 (severe).

\section{Immunohistochemical analysis}

The immunohistochemical analysis was performed using anti- $\alpha$-smooth muscle actin (anti $\alpha$-SMA) (EnvisionTM SystemHRP, Dako Cytomation, USA) visualized with diaminobenzidine. Positive and negative controls were included according to the manufacturer's guidelines. 
For the quantitative analysis of myofibroblasts, histological sections of $5 \mu \mathrm{c}$ of samples and immunohistochemical staining were performed. Images were taken of the tissue adjacent to the wound area. We used 10 surface fields (each with a field area of $0.024 \mathrm{~mm}^{2}$, totaling $0.24 \mathrm{~mm}^{2}$ ). Myofibroblasts quantification was performed by a single blinded observer. Myofibroblasts were considered to be either positive cells or brownish.

\section{Statistical analysis}

Statistical analysis was performed using GraphPad Prism Version 6.00 for Windows (La Jolla, California, USA, www. graphpad.com).

The expression of $\alpha$-smooth muscle actin in all groups and on all sampled days $(3,7,14$, and 21$)$, and the interaction between them was compared using a two-way ANOVA test.

Nonparametric variables were analyzed using a Mann-Whitney test. The significance level for rejecting the null hypothesis was $5 \%(\mathrm{p}<0.05)$.

\section{Results}

\section{Macroscopic and histological analysis}

No complications were reported in any animals during surgery. There were no deaths prior to euthanasia. Daily clinical evaluations showed adequate recovery rate, maintenance status, presence of physical activity, provision of food, and water intake in all groups. The diabetic animals daily showed signs of diabetes, with obvious weight loss despite increased food intake, and also had polyuria and polydipsia. The hair of the animals in this group was ruffled and yellowish.

The development of skin wounds in all animals showed oozing and delicate crusting up until the seventh day after surgery when the crust thickened and began to detach spontaneously as the healing process progressed.

Skin on the backs of the rats showed a pattern of free subcutaneous and very elastic loose tissue. Depending on the manipulation of the animal and the position in which they were immobilized for the purposes of measuring and photography, the size and appearance of the wounds changed significantly. We therefore considered this to be an unreliable way of analyzing the real effects of IGF-1 on the healing process.

In general, similar wound healing and histological patterns were observed in the animals that were treated with IGF-1 and those that were not. On the third postoperative day, an inflammatory process was observed, presenting congestion, hyperemia, necrosis, inflammatory cells, some collagen deposition, and absence of epithelium. On the seventh day post-surgery, congestion characteristic of the third day was still present, although it was less intense. The inflammatory process was more intense and diffused, with greater presence of inflammatory cells, and intense necrosis. Also on the seventh postoperative day, we observed the presence of new blood vessels (angiogenesis) and a larger number of fibroblasts that induced greater collagen neoformation (fibers were more numerous and more mature-looking) compared to the third day. Additionally, fibrin-leukocyte crusts were present in the wounds of all evaluated animals. On the 14th day after surgery, the wound healing process was advanced, showing a reduction in the inflammatory process characterized by a smaller amount of inflammatory cells, discrete inflammatory infiltration, absence of necrosis, re-epithelization of the tissues and collagen deposition, presenting more differentiated fibers (Figure 1). On the 21 st day, the wound healing process was

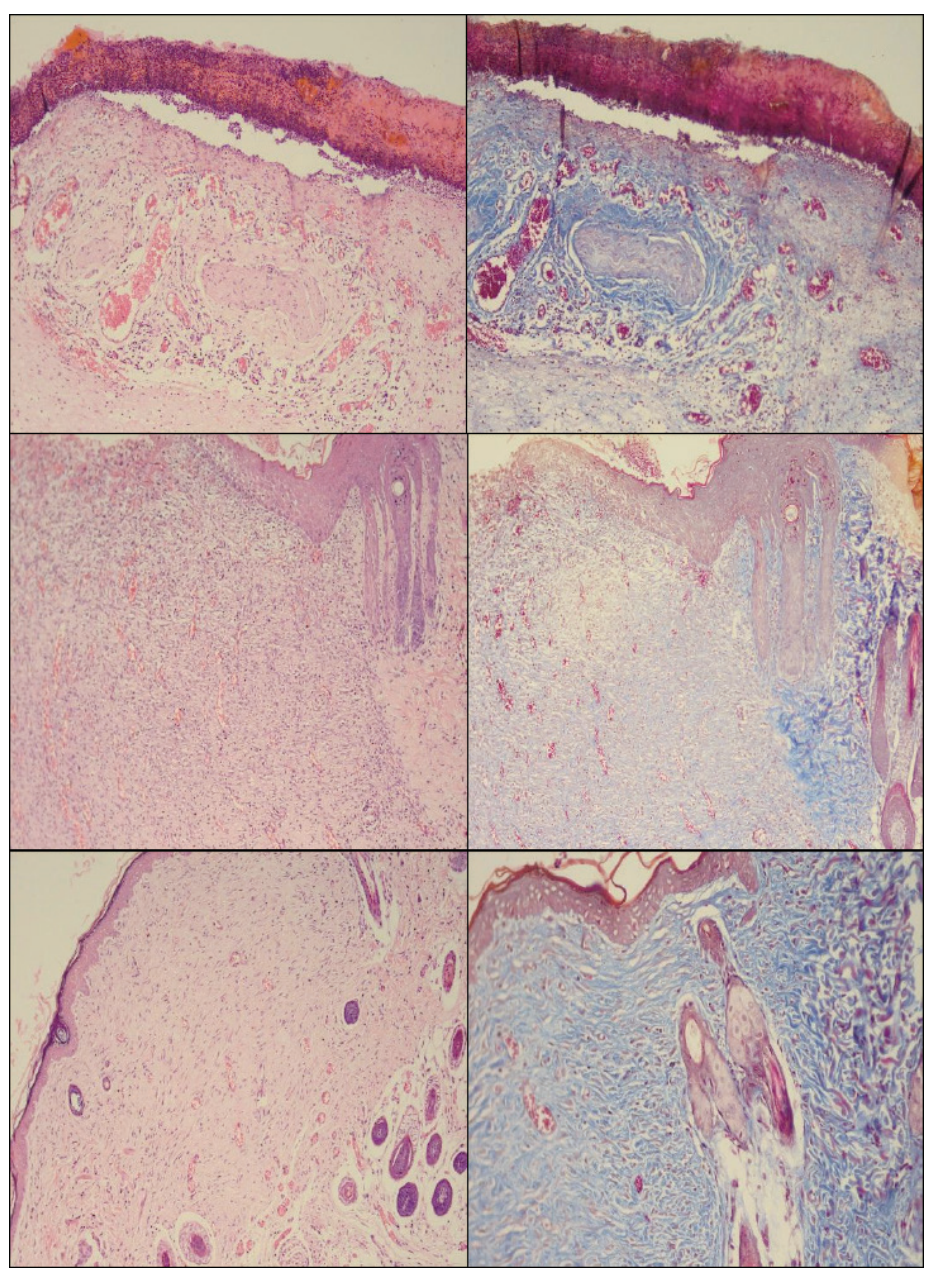

FIGURE 1 - Photomicrographs of the skin of non-diabetic, untreated rats at the $3 \mathrm{rd}, 7$ th and 21 st days after surgery. Hematoxicilina-Eosina and Masson's Tricromic staining. 
even more advanced, with a small amount of inflammatory cells and a completely reconstructed epithelium. By this point, granulation tissue was formed, characterized by intense collagen deposition (numerous and many mature aspect fibers).

Histological changes of the skin wounds were analyzed according to time after the injury and treatment, with the specific features of each stage recorded.

Regarding the acute inflammatory reaction, the results of the analysis of vascular proliferation and the mononuclear and polymorphonuclear analyses showed no statistically significant difference in any of the periods studied (according to the results of a Mann-Whitney test).

\section{Immunohistochemical analysis}

Tissue samples subjected to immunohistochemical analysis to compare the expression of $\alpha$-smooth muscle actin between groups showed a significant difference in the expression of myofibroblasts (Figure 2).

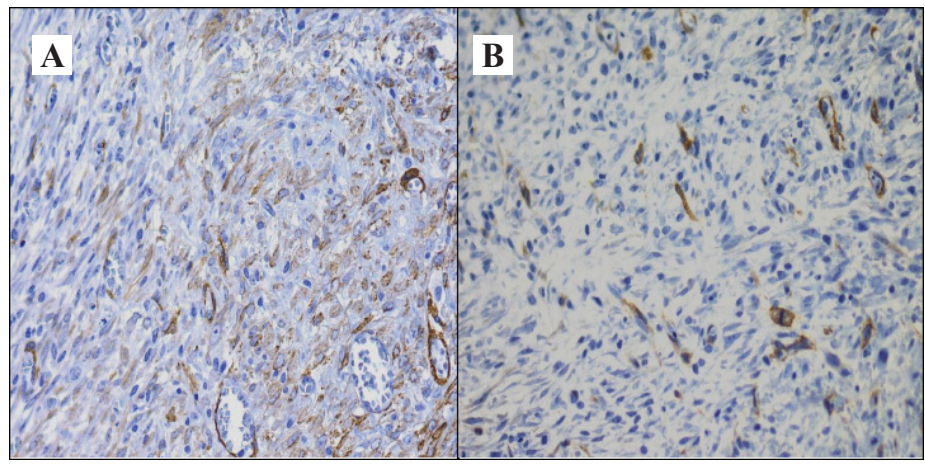

FIGURE 2 - (A) Diabetic, treated with 1\% IGF-1 and (B) Diabetic, untreated rats on the seventh day after surgery. Positive staining can be seen. Original magnification $\times 400$. (Vide original)

Figure 2 shows the expression of myofibroblasts cells stained brown in samples taken from diabetic animals treated with $3 \%$ IGF-1 in diabetic animals (left), and the photograph on the right shows the expression of myofibroblasts in tissue samples from diabetic animals that did not receive treatment.

The ANOVA test confirmed that these differences were statistically significant. The samples showed a peak in the expression of $\alpha$-smooth muscle actin ( $\alpha$-SMA) on the seventh day, which was higher in the groups treated with IGF. This difference was shown to be statistically significant.

Table 1 shows the average value of myofibroblasts in field 10 of the blades subjected to immunohistochemistry (Figure 2). In this table, one can see the significant presence of myofibroblasts on the seventh day after surgery, evident both in non-diabetic animals and in animals with diabetes that were treated with either $1 \%$ or $3 \%$ IGF- 1 .

TABLE 1 - Number of myofibroblasts in the wound area.

\begin{tabular}{ccccccc}
\hline \multicolumn{7}{c}{ DATA } \\
\hline DAYS & NDNT & NDIGF1 & NDIGF3 & DNT & DIGF1 & DIGF3 \\
\hline 3 & 0.0 & 0.0 & 0.0 & 0.0 & 0.0 & 0.0 \\
7 & 1.3 & 9.4 & 6.9 & 1.3 & 1.2 & 2.4 \\
14 & 0.2 & 0.0 & 1.2 & 0.0 & 0.0 & 1.2 \\
21 & 0.0 & 0.0 & 0.0 & 0.0 & 0.0 & 0.0 \\
\hline
\end{tabular}

Figures 3, 4 and 5 show the significant differences in expression of $\alpha$-smooth muscle actin in the skin wounds of diabetic and non-diabetic rats treated with IGF-1. The largest difference is seen on the seventh day in diabetics rats treated with IGF $3 \%$.

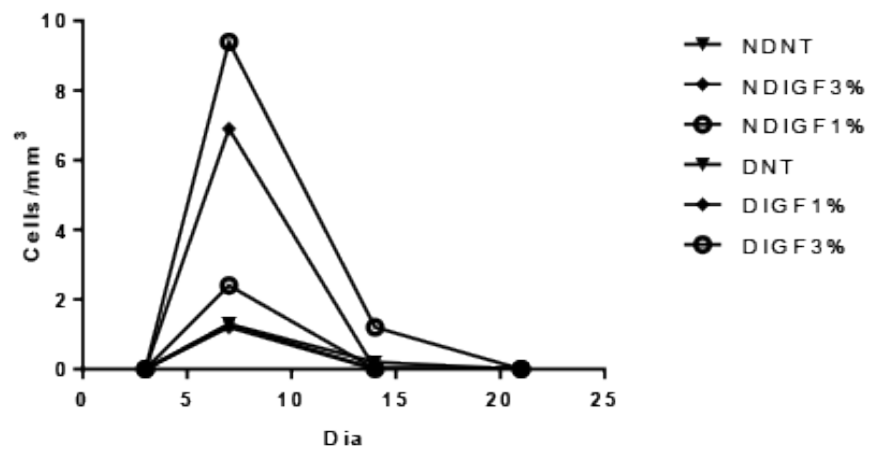

FIGURE 3 - Quantification of the expression of $\alpha$-smooth muscle actin in open wounds in diabetic and non-diabetic rats. ${ }^{*} \mathrm{p}=0.004(\mathrm{n}=4)$

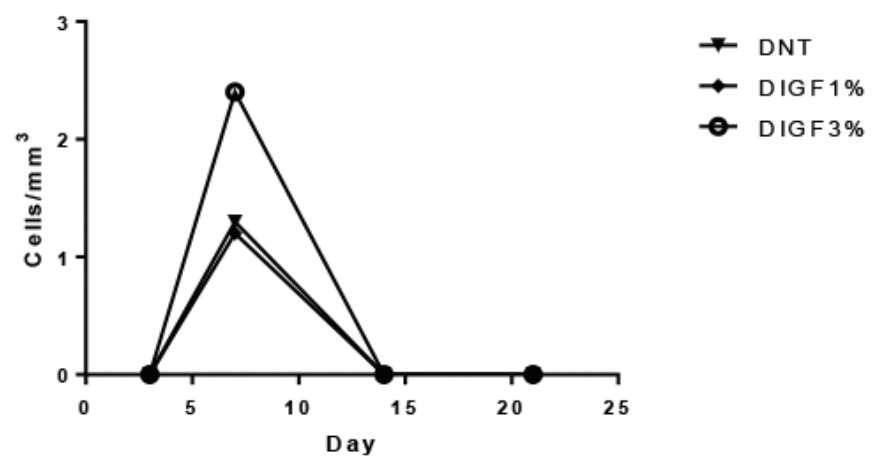

FIGURE 4 - Quantification of the expression of $\alpha$-smooth muscle actin in open wounds of diabetic rats. ${ }^{*} \mathrm{p}=0.002(\mathrm{n}=4)$ 


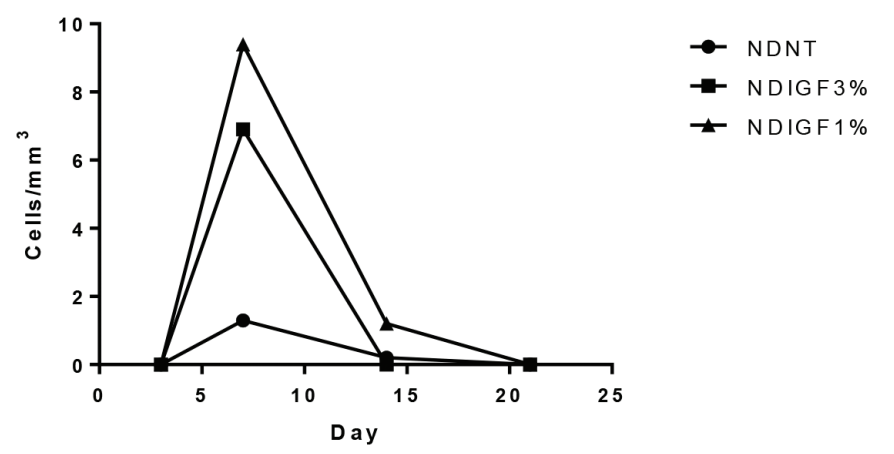

FIGURE 5 - Quantification of the expression of $\alpha$-smooth muscle actin in the open wounds of non-diabetic rats. ${ }^{*} \mathrm{p}=0.031(\mathrm{n}=4)$

\section{Discussion}

The aim of our study was to evaluate whether topical IGF-1 cream promotes an increase in tissue repair capacity and consequently in better wound healing in diabetic rats.

Several previous studies have demonstrated the expression of IGF-1 in the wounds of different species. Thus, IGF-1 was found in the wound fluid of rats and pigs ${ }^{12-14}$. In an attempt to identify IGFs and their receptors at the wound site, a group injury model used a freeze-thaw technique to study the expression of IGF-1 using immunohistochemistry $^{15}$. In normal skin, only a few cells in the dermis and epidermis express IGF-1. However, all epidermal cells, as well as macrophages and other cells, express IGF-1 at 1 to 3 days after the injury. Some other studies have suggested a role for the IGF system abnormalities associated with wound healing in animals with diabetes or treatment with glucocorticoids. Thus, one study found that diabetes induced in rats caused a $42 \%$ reduction in the levels of IGF-1 in the wound fluid and a $48 \%$ reduction in the plasma ${ }^{16}$.

Others have analyzed the expression of IGF-1 during wound healing in normal mice and genetically diabetic patients. They found that the mRNA expression of IGF-1 was severely retarded and reduced among diabetic patients ${ }^{6}$.

The specific effects of IGF in wound healing were studied, and it was demonstrated that IGF-1 plays a role in angiogenesis, promotion of collagen synthesis, and stimulation of fibroblasts and keratinocytes. IGF-1 has been found in the epithelium and in the later stages of granulation tissue. Increased expression of these growth factors is therefore related to changes in the wound ${ }^{3,6}$.

These data support the hypothesis that the difficulties associated with wound healing in diabetic patients are, at least in part, due to a deficiency in the activity of IGF-1 in the wound environment. These data are important to elucidate the pathogenesis of human chronic wounds, because IGF-1 proteins are absent in the basal layer of the epidermis and in fibroblasts from diabetic patients, but is present in healthy controls. In addition, IGF-1 proteins were shown to be absent in the basal layer of keratinocytes at the edge of the diabetic foot ${ }^{17}$.

The healing process involves the differentiation of fibroblasts into myofibroblasts. These cells have characteristic properties similar to those of smooth muscle cells, displaying the contractile phenotype through expression of $\alpha$-smooth muscle actin that promotes the approximation of the wound edges ${ }^{18}$.

Myofibroblasts synthesize extracellular matrix components such as collagen types I and III. During the normal healing of wounds, myofibroblasts disappear by undergoing apoptosis when epithelialization occurs. The transition from fibroblasts into myofibroblasts is influenced by mechanical stress and the presence of growth factors.

$\alpha$-Smooth muscle actin is a major myofibroblast differentiation marker and plays a role in regulating the expression of the collagen gene.

The role of $\alpha$-smooth muscle actin on the production of myofibroblasts in vitro has been previously demonstrated in experiments on wound healing in mice that used models involving fibroblasts ${ }^{19}$. Cells expressing this protein or differentiated myofibroblasts are strongly active compared with retractable protomiofibroblastos, in which there is an absence of any change in contractile protein expression ${ }^{20}$.

Our study found no statistically significant differences in the assessment of the healing process in the analysis of polymorphonuclear cells, vascularization, and inflammation. However, it did demonstrate the effectiveness of the application of IGF-1 in wound healing, which increases the expression of myofibroblasts. The immunohistochemical analysis using antibody $\alpha$-smooth muscle actin showed that the expression of myofibroblasts was more evident on the seventh postoperative day and was higher in the treated groups.

A significant increase was observed in the expression of myofibroblasts in the animals treated with IGF compared with those that were not treated. This increase occurred in both nondiabetic and diabetic animals, such that the expression in diabetic animals was even more significant than that in non-diabetic animals, suggesting that the receptors for this protein in diabetic animals may also be reduced. However, the increased availability helped to increase the expression of myofibroblasts, which in turn plays an important role in the final process of healing, promoting a more rapid healing process among treated animals.

Several authors have evaluated the use of numerous topical substances to positively influence the healing process. Obviously, many factors are involved in an effective intervention in the healing process, and accurate clinical diagnosis of the type 
of the injury and its healing stage is critical because different wounds in different patients require different forms of treatment.

In our study, IGF-1 cream was shown to be effective in the healing process; however, it is important to expand these analyses further. Future studies are needed to overcome the limitations of our experimental design as a whole and also to assess the expression of IGF-1 receptors in diabetic patients, other factors involved in wound healing, and thus evaluate the safety of treatment with IGF-1.

\section{Conclusions}

The use of a topical IGF-1 cream on wounds induced a higher expression of myofibroblasts in the wound bed and a consequent improvement in scarring.

In non-diabetic animals treated with IGF-1, the expression of myofibroblasts was higher than that in diabetic animals treated with IGF-1, which may indicate a decrease in IGF receptor expression in diabetic animals. Further studies are necessary to assess the decreased expression of these receptors.

\section{References}

1. Bruhn-Olszewska B, Korzon-Burakowska A, Gabig-Cimińska M, Olszewski P, Wegrzyn A, Jakóbkiewicz-Banecka J. Molecular factors involved in the development of diabetic foot syndrome. Acta Biochim Pol. 2012;59(4):507-13.

2. Abo El Asrar MA, Elbarbary NS, Elshennawy DE, Omar AM. Insulin-like growth factor-1 cytokines cross-talk in type 1 diabetes mellitus: relationship to microvascular complications and bone mineral density. Cytokine. 2012;59(1):86-93.

3. Werner S, Grose R. Regulation of wound healing by growth factors and cytokines. Physiol Rev. 2003;83(3):835-70.

4. Ching YH, Sutton TL, Pierpont YN, Robson MC, Payne WG. The use of growth factors and other humoral agents to accelerate and enhance burn wound healing. Eplasty. 2011;11:429-49.

5. Martin P. Wound healing-aiming for perfect skin regeneration. Science. 1997;276:75-81.

6. Brown Dl, Kane CD, Chernausek SD, Greenhalgh DG. Differential expression and localization of insulin-like growth factors I and II in cutaneous wounds of diabetic and nondiabetic mice. Am J Pathol. 1997;151(3):715-24.

7. Desmouliere A. Factors influencing myofibroblast differentiation during wound healing and fibrosis. Cell Biol Int. 1995;19(5):471-6.

8. Novosyadlyy R, Dudas J, Pannem R, Ramadori G, Scharf JG. Crosstalk between PDGF and IGF-1 receptors in rat liver myofibroblasts: implication for liver fibrogenesis. Lab Invest. 2006;86(7):710-23.

9. Kwiecinski M, Elfimova N, Noetel A, Töx U, Steffen HM, Hacker U,Nischt R, Dienes HP, Odenthal M. Expression of platelet-derived growth factor-C and insulin-like growth factor I in hepatic stellate cells is inhibited by miR-29. Lab Invest. 2012;92(7):978-87.
10. Hinz B, Phan SH, Thannickal VJ, Prunotto M, Desmoulière A, Varga J, De Wever O, Mareel M, Gabbiani G. Recent developments in myofibroblast biology: paradigms for connective tissue remodeling. Am J Pathol. 2012;180(4):1340-55.

11. Lerco MM, Spadella CT, Machado JLM, Schellini SA, Padovani CR. Experimental alloxan diabetes-induced: a model for clinical and laboratory studies in rats. Acta Cir Bras. 2003;18:132-42.

12. Marikovsky M, Vogt P, Eriksson E, Rubin JS, Taylor WG, Joachim $\mathrm{S}$, Klagsbrun M. Wound fluid-derived heparin-binding EGF-like growth factor (HB-EGF) is synergistic with insulin-like growth factor-I for Balb/MK keratinocyte proliferation. J Invest Dermatol. 1996;106(4):616-21.

13. Robertson JG, Belford DA, And Ballard FJ. Clearance of IGFs and insulin from wounds: effect of IGF-binding protein interactions. Am J Physiol. 1999;276(4):663-71.

14. Steenfos HH, Jansson JO. Gene expression of insulin-like growth factor-I and IGF-1 receptor during wound healing in rats. Eur J Surg. 1992;158(6-7):327-31.

15. Jennische E, Skottner A, Hansson HA. Dynamic changes in insulinlike growth factor I immunoreactivity correlate to repair events in rat ear after freeze-thaw injury.Exp Mol Pathol. 1987;47(2):193-201.

16. Bitar MS, Labbad ZN. Transforming growth factor-beta and insulinlike growth factor-I in relation to diabetes-induced impairment of wound healing. J Surg Res. 1996;61(1):113-9.

17. Blakytny R, Jude EB, Martin Gibson J, Boulton AJ, Ferguson MW. Lack of insulin-like growth factor 1 (IGF1) in the basal keratinocyte layer of diabetic skin and diabetic foot ulcers. J Pathol. 2000;190(5):589-94.

18. Mandelbaum SH, Di Santis EP, Mandelbaum MHS. Cicatrização: conceitos atuais e recursos auxiliares. An Bras Dermatol. 2003;78(4):393-410.

19. Hinz B, Celetta G, Tomasek JJ, Gabbiani G, Chaponnier C. Alphasmooth muscle actin expression upregulates fibroblast contractile activity. Mol Biol Cell. 2001;12(9):2730-41.

20. Bogatkevich GS, Tourkina E, Abrams CS, Harley RA, Silver RM, Ludwicka-Bradley A. Contractile activity and smooth muscle alpha actin organization in thrombin-induced human lung myofibroblasts. Am J Physiol Lung Cell Mol Physiol. 2003;285(2):334-43.

\section{Correspondence:}

José Lucio Martins Machado

Rua Cesário Galeno, 448/475

03071-000 São Paulo - SP Brasil

Tel.: (55 11)2178-1252

jluciomm@yahoo.com.br

Received: Oct 23, 2013

Review: Dec 20, 2013

Accepted: Jan 21, 2014

Conflict of interest: none

Financial source: Scientific Initiation Project Grant - PIBIC/CNPq

${ }^{1}$ Research performed at Laboratory of Experimental Surgery, Sao Paulo City University (UNICID), Sao Paulo-SP, Brazil. Part of Master degree thesis, Postgraduate Program in Health Sciences, Sao Paulo State Public Servant Hospital (IAMSPE). Tutor: Prof. Dr. José Lucio Martins Machado. 